\title{
Utility of Cell and Molecular Biology Research for the Diagnosis and Therapy of Oral Premalignant Lesions
}

\author{
Doinița Temelie-Olinici ${ }^{1}$, Carmen-Elena Cotrutz ${ }^{1 *}$, Ana Emanuela Botez ${ }^{1 *}$, Laura Gheucă- \\ Solovăstru ${ }^{2}$, Vasile Bogdan Grecu ${ }^{1}$, Laura Stoica ${ }^{1}$ and Oana Mihaela Condurache Hritcu ${ }^{1}$ \\ ${ }^{1}$ Department of Morpho-Functional Sciences II, Grigore T Popa, România \\ ${ }^{2}$ Departement of Dermatology, Grigore T Popa, România
}

\begin{abstract}
Introduction: Premalignant oral lesions represent one of the most important risk factors for the development of oral scuamoous cell carcinoma, the sixth most common malignancy diagnosed worldwide. The characteristic phenotypic and molecular variability of these lesions condition the early identification of the risk of malignant transformation, with a significant impact on prognosis and quality of life. Accurate and complete description of cell interactions involved in the carcinogenic cascade requires the use of the results of cell and molecular biology research. In this regard, this paper aims to evaluate the intricate role of molecular markers in assessing the progression of the most common premalignant oral lesions.

Materials and Methods: To achieve these aims, oral mucosal fragments were collected from 53 patients diagnosed with leukoplakia, erosive oral lichen planus and erosive actinic cheilitis. After an anatomopathological diagnosis was made, the tissue fragments were processed by immunohistochemical and ELISA techniques to perform a correlation and comparative study of ezrin, interleukin- 6 (IL-6) and cyclooxygenase 2 (Cox-2).

Results: IL-6 and Cox-2 levels were higher in premalignant lesions than in normal oral mucosal epithelium. Ezrin immunoreactivity was more intense at cytoplasmic level in premalignant lesions compared to normal mucosa, where expression was only identified at membrane level. Discussion: Intensity of ezrin immunopositivity also correlates with increased IL- 6 expression, revealed by the biochemical study, indicating the influence on NF- $\kappa B$ signaling pathway. Cytoplasmic ezrin overexpression is associated with elevated Cox-2 levels in dysplastic lesions.
\end{abstract}

Conclusions: The identification and characterization of the molecular profile of premalignant oral lesions allows their correct and complete staging and the improvement of personalized therapeutic management.

KEYWORDS: Oral premalignant lesion; Oral cancer; Biomarkers; Ezrin; Interleukin-6; Cyclooxygenase-2

\section{INTRODUCTION}

In the past twenty years, multiple population studies have highlighted the significant increase in incidence and prevalence of premalignant oral lesions, precursors of oral scuamous cell carcinoma. In addition, their malignant transformation rate is estimated at $18-25 \%$ within a variable interval of 7-14 years after diagnosis [1-5]. The morpho functional characteristics of the oral mucosa, constantly exposed to the action of various proinflammatory environmental, internal and external factors, explain its susceptibility to infectious/inflammatory processes,

Quick Response Code:

Address for correspondence: Carmen Elena Cotrutz, Professor, MD, PhD, Department of Morpho-Functional Sciences II, Grigore T Popa, University of Medicine and Pharmacy, Romania

Ana Emanuela Botez, Assistant Professor, MD, Department of Morpho-Functional Sciences II, Grigore T Popa, University of Medicine and Pharmacy, Romania

Received: September 15, $2020 \quad$ Published: October 05, 2020

How to cite this article: Doinița TO, Carmen EC, Ana EB, Laura GS, Vasile BG, Laura S, Oana Mihaela CH. Utility of Cell and Molecular Biology Research for the Diagnosis and Therapy of Oral Premalignant Lesions. 2020 - 2(5) OAJBS.ID.000221. DOI: 10.38125/OAJBS.000221 
creating a predilect environment for the development of numerous premalignant and malignant lesions, with a special impact on morbidity and mortality identified in the general population $[1,2]$. Over the years, the results of cell and molecular biology research have shown the regulatory role of such pro- inflammatory molecules as interleukin-6 (IL-6) and cyclooxygenase-2 (COX-2) on epithelial-mesenchymal transition (EMT), critical event in malignant transformation. Thus, the hypothesis that cancer arises from inflammatory sites (commonly referred to as "lymphoreticular infiltration") is supported. In addition, elevated levels of prostaglandin E2 (PGE2), a metabolite produced by the catalytic action of Cox-2, are associated with an increased risk of invasiveness and angiogenesis since the early stages of carcinogenesis [3-6].

A number of recent studies highlight the role of ezrin, a component of the ERM (Ezrin/ Radixin/Moesin) complex, in regulating cell signaling pathways controlling survival, proliferation and cell migration. These pathological implications have led to the proposed use of ezrin as a biomarker in establishing the therapeutic strategy for head and neck carcinomas [7-10].

Given that the diagnostic and therapeutic management recommended by numerous national and international guidelines is significantly limited by the complexity of the etiopathogenic mechanisms characteristic to pre-malignant oral lesions, the present study aims to characterize some molecular markers responsible for the modification of cell interactions/signaling pathways specific to these lesions and their subsequent progression, drawing attention to the essential relationships both between these markers and between their expression and clinical-therapeutical aspects.

\section{MATERIALS AND METHODS}

To attain the desired goals, oral mucosal fragments were collected by incisional/excisional biopsy with the scalpel blade from 53 females, non-smokers, non- chronic alcohol users, aged 45-60 years, and clinically and histopathological diagnosed with: leucoplakia - 28 cases, of which 14 cases had mild-to-moderate dysplasia and 14 cases moderate-to-severe dysplasia; actinic erosive cheilitis - 10 cases; erosive oral lichen planus - 15 cases. At the same time, normal oral mucosal fragments were collected by the same technique from perilesional areas and served as a control group. Prior to surgery, informed consent was obtained from each patient, in accordance with the legislation in force. The anatomopathological diagnosis was made at the Mortuary and Anatomic Pathology Unit of the Iasi Railroad Hospital, and Pathological Anatomy Laboratory of the Iasi "Dr. I Czihac "Military Emergency.

The tissue samples collected from the study patients were divided into several fragments, part of them being paraffinembedded, then sectioned and stained with haematoxylin-eosin in view of making an anatomopathological diagnosis, while others were either dewaxed for use in the ELISA technique or processed by the immunohistochemical technique using the Novo Link TMMax Polymer Detection System kit. The microscopic preparations were examined with an Olympus BX40 microscope with attached Olympus E330 camera at the Department of Cell and Molecular Biology of the Iasi University of Medicine and Pharmacy.

\section{RESULTS}

By using the ELISA technique, we identified at tissue level increased IL-6 and Cox-2 levels in the samples collected from premalignant lesions compared to normal tissue (Table 1). IL-6 levels were higher in leukoplakia lesions with moderate-severe dysplasia than in the other premalignant lesions studied. The lowest IL-6 concentration was found in the normal oral mucosa (Table 1). Cox- 2 was present in low concentrations in the normal oral mucosa. Relative to premalignant lesions, the highest concentrations were found in both leukoplakia types, and the lowest in erosive actinic cheilitis (Table 1). Examination of normal oral mucosa samples processed by the immunohistochemical technique revealed a moderately positive peri membranous ezrin immunoreactivity and absence of ezrin immunoreactivity at cytoplasmic level (Figure 1).

Compared to these findings, the examination of the samples obtained from leuloplakia tissue lesions revealed a strongly positive intracytoplasmic immunoreactivity even within the superficial parakeratotic layer along with perimembranous granular immunoreactivity, with a series of differences between the two types of leukoplakia in view. Thus, in less severe leukoplakia forms associated with mild dysplasia there was an intense, granular, peri membranous and intracytoplasmatic immunopositivity up to the superficial parakeratotic layer (Figure 2) compared to a much more attenuated reaction at membrane level but intensely positive intracytoplasmatically in the forms of severe leukoplakia associated with severe dysplasia lesions (Figure 3).

Table 1: Tissular expression of IL-6 and Cox-2 (Medium values).

\begin{tabular}{|c|c|c|c|}
\hline Sample & \multirow{2}{*}{} & IL-6 & Cox-2 \\
\cline { 3 - 4 } & 14 & (ng/mg protein) & 2.98 \\
\hline $\begin{array}{c}\text { Leukoplakia with easily } \\
\text { moderated dysplasia }\end{array}$ & 14 & 0.016 & 3.71 \\
\hline $\begin{array}{c}\text { Leukoplakia with severely } \\
\text { moderated dysplasia }\end{array}$ & 10 & 0.028 & 2.37 \\
\hline Erosive cheilitis & 15 & 0.017 & 2.85 \\
\hline Oral erosive lichen planus & 53 & 0.022 & 0.54 \\
\hline Oral mucosa witness & & 0.004 & \\
\hline
\end{tabular}

In erosive oral lichen planus cases, the examination of tissue samples revealed a cytoplasmic point-and- point immunopositivity and in some cases perinuclear drop-shaped in the basal and malpighian layers (Figure 4). In the cases diagnosed with erosive actinic cheilitis, immunohistochemistry revealed membranous and moderately cytoplasmic ezrin immunopositivity in the basal and malpighian layers (Figure 5).

\section{DISCUSSION}

Currently, the mechanisms by which acute and chronic inflammation induced by various bacterial, viral, fungal, chemical and physical factors triggers and sustains the oral carcinogenic cascade is not fully understood. Knwon is only the ability of tumor cells to secrete a number of inflammatory factors which modify the stroma and facilitate invasion $[11,12]$. 
IL-6, Th2 cytokine (Thelper 2) is considered a tumor marker with a sensitivity of $60-70 \%$ and a specificity of 58-90\%. However, the available studies used to define the diagnostic and prognostic value of this molecule are few and controversial [3,13-15]. IL-6 activity is frequently related to the concept of "epigenetic field" existing in the oral cavity, although the idea that IL-6-mediated suppression of signaling or inhibition of chronic inflammation influence the epigenetic changes is not supported. In dysplastic lesions, the increase in IL-6 level is directly proportional to the histological degree of dysplasia $[5,7,11]$ as also shown by Table 1 .

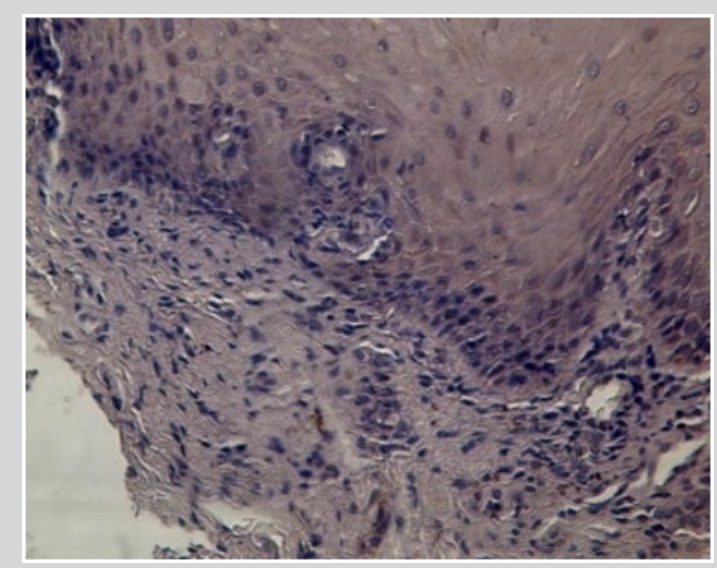

Figure 1: Normal oral mucosa.

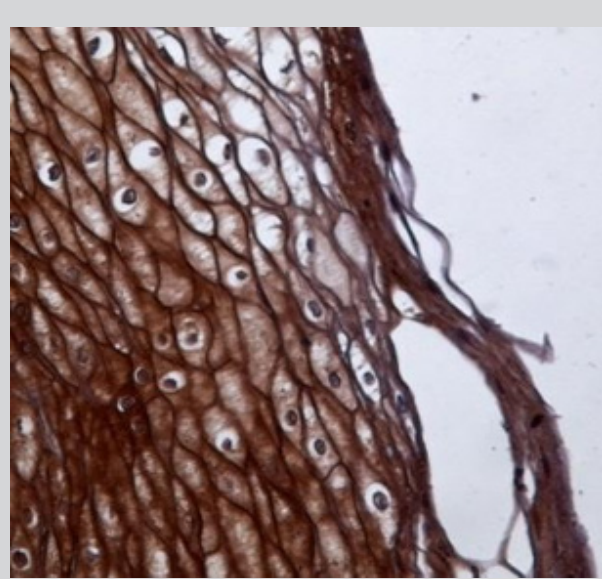

Figure 2: Leukoplakia with mild-moderate dysplasia IHE 40x.

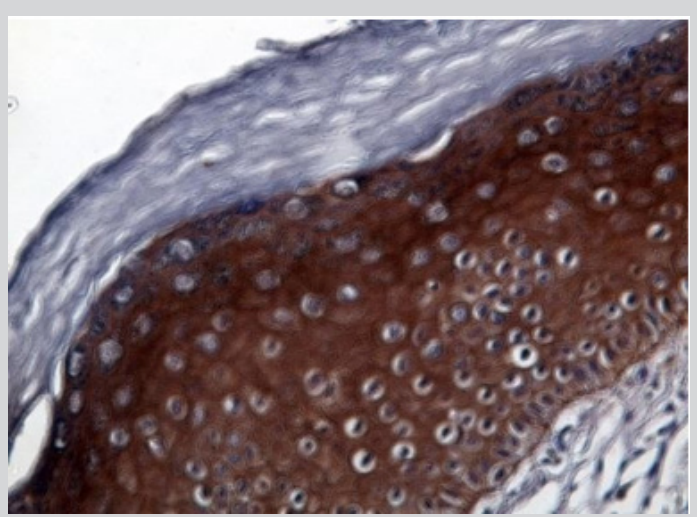

Figure 3: Leukoplakia with mild-severe dysplasia. IHE; 40x.
In oral squamous cell carcinomas, Il-6 acts as an immunosuppressive molecule that facilitates the "evasion" of immune system control mechanisms. Thus, in cancer patients, elevated serum IL-6 levels are negatively correlated with prognosis [14]; by extrapolation, a high IL-6 level detected by ELISA technique in our premalignant lesion cases suggests a high degree of aggressiveness of some of the studied lesions and a marked tendency to malignant transformation $[16,17]$.

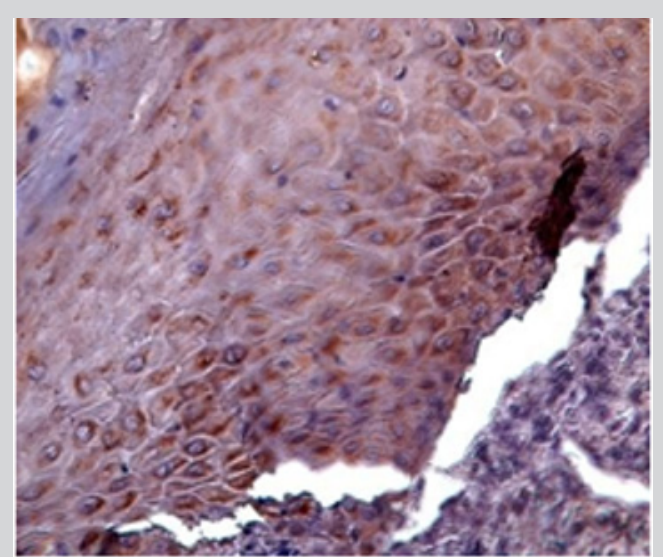

Figure 4: Erosive oral lichen planus. IHE; 40x.

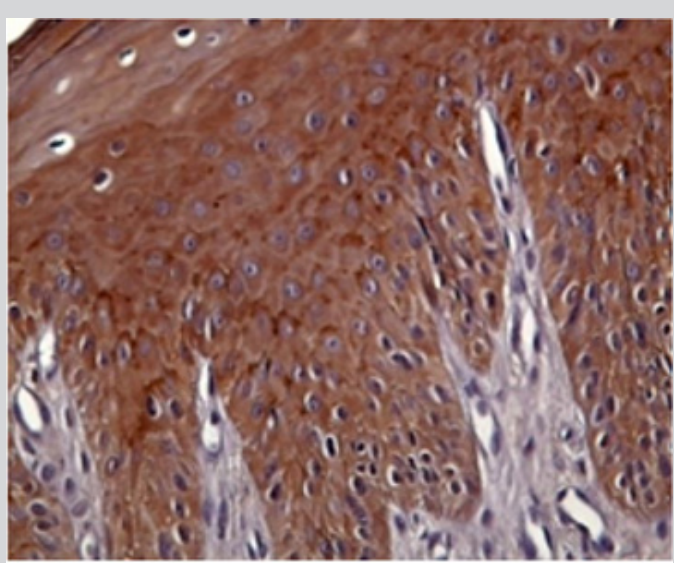

Figure 5: Erosive actinic cheilitis. IHE; 40x.

Also, some studies indicate that, in line with the increased production of inflammatory mediators, this cytokine is on the one hand stimulated early in the premalignant lesions prior to their transformation into carcinoma, and, on the other hand, it is more prominent in premalignant than in malignant lesions [15-19]. In the present study we detected 4 to 7 -fold higher IL-6 levels in the premalignant lesions compared to normal tissue. The presence of IL-6 in normal tissue fragments may confirm the presence of a chronic inflammatory process induced by factors other than those identified in the statistical studies conducted so far.

Goel 2015 suggested that IL-6 plays a role as a growth factor, suggesting its importance in cancer development and progression. Moreover, they believe that Il-6 serum and/or salivary level may be an indicator of chemotherapy efficacy [20-21].

In erosive lichen planus, IL-6 controls the proliferation and differentiation of cytotoxic $\mathrm{T}$ lymphocytes, with destructive effects on epithelium, and induces apoptosis of undifferentiated keratinocytes [19]. The results of this study are consistent with those reported by [20]. Who identified increased IL-6 expression in both oral and cutaneous lichen planus lesions? Thus, the stimulating 
action of IL-6 on keratinocyte proliferation is suggested, explaining the hyperplastic changes detected in lichen planus (Table 1).

In erosive actinic cheilitis, lesions IL-6 level was lower than in leukoplakia lesions with moderate-severe dysplasia and erosive oral lichen planus, and insignificantly higher than that detected in leukoplakia with a mild- to-moderate dysplasia. These results may confirm the implication in ultraviolet-B (UVB) radiation in the etiopathogenesis of cheilitis, hypothesis proposed in 1923 [21].

Cox-2 expression is stimulated by mutagenic and proinflammatory factors, such as cytokine IL-6, dependent on $\mathrm{NF}-\kappa \mathrm{B}$ activation, the results of this study supporting this finding $[13,16,18]$. Increased IL- 6 and Cox-2 levels identified in erosive actinic cheilitis lesions can be causally related to exposure to UVB, radiation that stimulates both Cox- 2 and NF-kB expression [16]. Understanding these processes is crucial as it can lead to the identification of new forms of treatment. Imiquimod, a topical preparation commonly used in the treatment of oral premalignant lesions can stimulate IL- 6 production, thus explaining such side effects as aphthous mouth ulcer [5,17]. Currently a number of phase I/II clinical trials suggest the use of anti-IL-6 or IL-6R antibodies as therapeutic alternatives. However, the fact that the association of IL-6 with multidrog resistance proteins (MRPs) expressed by keratinocytes of the oral mucosa epithelium plays an important role in tumor pathogenesis should not be overlooked. This account, among others, for the resistance to methotrexate therapy in patients with lichen planus $[3,19]$.

It is well known that both radiation therapy and chemotherapy can induce biosynthesis of Cox- 2 and prostaglandins with potential therapeutic implications [20]. Numerous experimental studies investigate the role of Cox-2 inhibitorsin suppressing carcinogenesis. In addition to genetic evidence, numerous pharmacological studies suggest or indicate Cox-2 as a therapeutic target. For example, Subbaramaiah and Dannenberg demonstrate that selective Cox2 inhibitor therapy reduces the formation of tongue, oesophagus, breast, skin, lung and bladder tumors. However, the regression of these tumors is very rare. Therefore, these inhibitors can be used as adjuvant therapy $[21,22]$.

The transition from dysplasia to invasive cancer is one of the most important stages of carcinogenesis involving numerous biological processes, basement membrane degradation by proinflammatory factors included [22]. Given our goal to identify the molecules that influence the development of some signaling pathways and/or signal transduction mechanisms involved in malignant transformation as well as for the early detection of malignant transformation at the level of oral structures, we initiated an immunohistochemical study of ezrin, a molecule very little exploited in literature and research centres. There are two forms of ezrin: active, physiologically located in the membrane, and inactive, identified in the cytoplasm [23,24]. In the cytoplasm, ezrin, similar to the other proteins of the ERM complex, is expressed as "dormant" monomers in which F-actin and plasma membrane binding sites are masked. Moreover, it has a closed conformation due to intramolecular $\mathrm{N}$-terminal-C-terminal association. Their activation requires

destroying the association. Two factors are known for their involvement in activation: PIP2 binding and phosphorylation of a threonine residue at the C-terminal end [25]. These aspects correlated with the results of the present study indicating the perimembranous ezrin expression normal oral mucosa and absence of immunoreactivity to this molecule at cytoplasmic level (Figure 1) indicate its functional role in the organization of cellular coupling and membrane domains specific to each type of tissue. The presence of intense cytoplasmic immunoreactivity in erosive lichen planus and leukoplakia associated with moderate-severe dysplasia (Figure 3,4) suggests a change in epithelial cell molecular phenotype, activation of cytoplasmic "dormant" monomers, that is triggering of some phosphorylation reactions at this level and also a clear tendency to malignanization and an aggressive malignant phenotype, having in view the literature data showing that cytoplasmic localization in head and neck squamous cell carcinoma is associated with poor prognosis and reduced survival rate. In the examined erosive actinic cheilites cases, membrane immunopositivity was identified in all layers and discretely cytoplasmic in the spinous layer (Figure 5).

Recent studies describe the ability of the ERM protein complex to interact with transmembrane proteins, phospholipids, cytoplasmic membrane-associated proteins, and cytoskeleton, being involved in numerous physiological processes, including preservation of cell shape, motility, proliferation and development [26-28].

This gives ezrin a key role in organizing specific membrane domains, essential areas in intercellular signaling. The mechanism responsible for the cytoplasmic location of ezrin and the way cytoplasmic ezrin contributes to the aggressive phenotype of squamous cell carcinoma is not fully understood [27]. The results of the biochemical study showed that the intensity of ezrin immunosuppression also correlates with increased IL-6 expression indicating that ezrin influences the NF- $\mathrm{KB}$ mediated signaling pathway, and the association of cytoplasmic ezrin overexpression with increased Cox- 2 levels in dysplastic lesions suggests activation of protein kinase $\mathrm{C}$.

\section{CONCLUSION}

Expression of is associated with both malignant transformation and severity of squamous cell carcinoma. Starting from the clinical findings according to which, despite invasive surgical management, premalignant dysplastic lesions are a risk factor for the development of squamous cell carcinoma, the present research supports the need for clinical trials able to establish a correct therapeutic management based on a complete molecular and morphological profile; this way, the risk of recurrences and the side-effects associated with current therapies can be limited.

\section{REFERENCES}

1. Unver N, Delgado O, Zeleke K (2018) Reduced IL-6 levels and tumor-associated phospho-STAT3 are associated with reduced tumor development in a mouse model of lung cancer chemoprevention with myoinositol. Int J Cancer 142(7): 1405-1417.

2. Sahingur SE, Yeudall WA (2015) Chemokine function in periodontal disease and oral cavity cancer. Front Immunol 6(214): 1-15.

3. Dinesh KT, Ashwini BK, Rameshkumar A (2016) Salivary and serum interleukin 6 levels in oral premalignant disorders and squamous cell carcinoma: diagnostic value and clinicopathologic correlations. Asian Pac J Cancer Prev 17(11): 4899-4906.

4. Kamperos G, Nikitakis N, Sfakianou A (2016) Expression of NF- $\kappa B$ and IL- 6 in oral precancerous and cancerous lesions: An immunohistochemical study. Med Oral Patol Oral Cir Bucal 21(1): e6-13.

5. Parakh MK, Ulaganambi S, Ashifa N (2019) Oral potentially malignant disorders: Clinical diagnosis and current screening aids: a narrative review. Eur J Cancer Prev 8(1): 1-9.

6. Cassol-Spanemberg J, Rodríguez-de Rivera-Campillo ME, Otero-Rey EM (2018) Oral lichen planus and its relationship with systemic diseases. A review of evidence. J Clin Exp Dent 10(9): e938-e944. 
7. Ai R, Tao Y, Hao Y (2017) Microenvironmental regulation of the progression of oral potentially malignant disorders towards malignancy. Oncotarget 8(46): 81617-81635.

8. Zheng X, Mao C, Qiao H (2017) Plumbagin suppresses chronic periodontitis in rats via down- regulation of TNF- $\alpha$, IL- $1 \beta$ and IL- 6 expression. Acta Pharmacol Sin 38(8): 1150-1160.

9. Ara T, Nakatani S, Kobata K, Sogawa N, Sogawa C (2018) The biological efficacy of natural products against acute and chronic inflammatory diseases in the oral region. Medicines (Basel) 5(4): 1-9.

10. Tampa M, Mitran MI, Mitran CI, Sarbu MI (2018) Mediators of inflammation - A potential source of biomarkers in oral squamous cell carcinoma. J Immunol Res 2018: 1-12.

11. Olinici D, Cotrutz CE, Mihali CV (2018) The ultrastructural features of the premalignant oral lesions. Rom J Morphol Embryol 59(1): 243-248.

12. Samimi M (2016) Cheilitis: Diagnosis and treatment. Presse Med 45(2): 240-250.

13. Kakkar VV, Saini MK, Kaur IP (2019) Nano drug delivery in treatment of oral cancer: A review of the literature. Curr Drug Targets 20(10): 10081017.

14. Warnakulasuriya S (2018) Clinical features and presentation of oral potentially malignant disorders. Oral Surg Oral Med Oral Pathol Oral Radiol 125(6): 582-590

15. Awadallah M, Idle M, Patel K, Kademani D (2018) Management update of potentially premalignant oral epithelial lesions. Oral Surg Oral Med Oral Pathol Oral Radiol 125(6): 628-636.

16. Akbarzadeh BA, Taghavi N, Shahla M (2017) Combined analysis of vascular endothelial growth factor expression with cyclooxygenase- 2 and mast cell density in oral squamous cell carcinoma. Pathobiology 84(2): 80-86.

17. Sinanoglu A, Soluk-Tekkesin M, Olgac V (2015) Cyclooxygenase-2 and Ki67 expression in oral leukoplakia: A clinicopathological study. J Oral Maxillofac Res 6(2): e3-e13.
18. Seyedmajidi M, Shafaee S, Siadati S (2014) Cyclooxygenase-2 expression in oral squamous cell carcinoma. J Cancer Res Ther 10(4): 1024-1029.

19. Panneer SN, Sadaksharam J (2015) Salivary interleukin-6 in the detection of oral cancer and precancer. Asia Pac J Clin Oncol 11(3): 236-241.

20. Bonavida B, Baritaki S (2017) The novel role of Yin Yang 1 in the regulation of epithelial to mesenchymal transition in cancer via the dysregulated NF-кB/Snail/YY1/RKIP/PTEN Circuitry. Crit Rev Oncog 16(3-4): 211-226.

21. Bagan L, Sáez GT, Tormos MC (2016) Salivary and serum interleukin-6 levels in proliferative verrucous leukoplakia. Clin Oral Investig 20(4): 737-743.

22. Kakurina GV, Kondakova IV, Spirina LV (2018) Expression of genes encoding cell motility proteins during progression of head and neck squamous cell carcinoma. Bull Exp Biol Med 166(2): 250-252.

23. Eckert AW, Wickenhauser C, Salins PC (2016) Clinical relevance of the tumor micro-environment and immune escape of oral squamous cell carcinoma. J Transl Med 14(1): 85-93.

24. Chen MJ, Gao XJ, Xu LN (2014) Ezrin is required for epithelial-mesenchymal transition induced by TGF- $\beta 1$ in A549 cells. Int J Oncol 45(4): 1515-1522.

25. Olinici D, Gheucă-Solovăstru L, Stoica L (2016) The molecular mosaic of the premalignant cutaneous lesions. Rom J Morphol Embryol 57(2): 353-359.

26. Li J, Wei K, Yu H (2015) Prognostic value of ezrin in various cancers: a systematic review and updated meta-analysis. Sci Rep 5: 1-13.

27. Mohanraj R, Ramani P, Premkumar P (2017) Immunohistochemical expression of ezrin in oral potentially malignant disorders- A descriptive study. J Pharm Bio-allied Sci 9(Supple 1): S205-S210.

28. Fröse J, Chen MB, Hebron KE (2018) Epithelial-mesenchymal transition induces podocalyxin to promote extravasation via ezrin signaling. Cell Rep 24(4): 962-972. 